\title{
Padrões Emergentes em Redes Cerebrais e Redes Sociais e suas Influências na Cognição Humana
}

\author{
Viviane Soares Rodrigues Silva ${ }^{1}$, Claudia Lage Rabello Motta ${ }^{2}$, José Otávio \\ Pompeu e Silva ${ }^{2}$
}

${ }^{1}$ Programa de Pós Graduação em Informática - Universidade Federal do Rio de Janeiro [PPGI/UFRJ] - Rio de Janeiro - RJ - Brasil

${ }^{2}$ Instituto Tércio Pacitti de Aplicações e Pesquisas Computacionais - Universidade Federal do Rio de Janeiro [NCE/UFRJ] - Rio de Janeiro - RJ - Brasil

vivianerodrig@ppgi.ufrj.br, \{claudiam, pompeuesilva\}@nce.ufrj.br

Abstract. The human brain can be structured and understood through the dynamic relationships of a network, by using the Graph Theory. Recent studies have showed that topological patterns from functional connections of brain networks might be associated with the human intellectual performance. On the other hand, the same Graph Theory tools can also be used to construct the network social architecture and to understand the emergent phenomena from relationships between people. This article presents the hypothesis about the correlation between interpersonal behavior patterns, mapped as a social network, and intracerebral relationships patterns, mapped as a brain network.

Resumo. O cérebro humano pode ser estruturado e compreendido através das dinâmicas relações de uma rede, utilizando a Teoria dos Grafos. Estudos recentes mostram que padrões topológicos resultantes das conexões funcionais das redes cerebrais podem estar relacionados ao desempenho intelectual humano. Por outro lado, as mesmas ferramentas da Teoria dos Grafos também podem ser empregadas para se compreender a arquitetura e fenômenos emergentes das relações sociais estabelecidas entre as pessoas. Este artigo traz a hipótese de que possa existir alguma correlação entre padrões de comportamento das relações interpessoais mapeadas como uma rede social e as relações intracerebrais, mapeadas como uma rede cerebral.

\section{Introdução}

A ciência se preocupa com a estrutura, comportamento e evolução de sistemas complexos como células, cérebro, ecossistemas, sociedade ou economia global. Para entender estes sistemas não basta o conhecimento sobre os elementos que compõem cada sistema, é preciso saber como estes elementos interagem entre si e que propriedades emergem destas interações. Tais interações formam um emaranhado de conexões que, por sua vez, seguem padrões inerentes a cada sistema em questão. Esta conectividade apresenta-se em diversas formas: interações moleculares, conexões sinápticas, associações semânticas, teia alimentar, redes sociais, hiperlinks da internet, entre autores quando escrevem e publicam trabalhos em coautoria, ou nas citações que fazem de um trabalho ao outro [Sporn, 2011]. 
O conjunto das conexões dos elementos de um dado sistema pode ser representado como uma rede, sendo cada elemento um vértice desta rede, e existirá uma aresta entre estes vértices caso possuam o tipo de relação definido para a construção desta rede [Newman, 2010].

Em particular, o cérebro é um sistema complexo, composto por um grande número de elementos neurais interconectados por sinapses e caminhos axomáticos, por onde a informação é constantemente processada e integrada entre regiões especializadas, espacialmente distribuídas mas funcionalmente conectadas [Sporns et al, 2000]. A utilização de métodos quantitativos pertencentes à Teoria dos Grafos podem comprovar esta configuração anatômica do cérebro [Sporns et al., 2004; van den Heuvel et al., 2009a]. Esta integração da informação em nosso cérebro permite-nos avaliar o mundo ao nosso redor e responder a situações complexas [van den Heuvel, 2009b]. Alterações nos estímulos sensoriais ou tarefas cognitivas resultam num padrão de atividade cerebral altamente especializado e o resultado da análise destes padrões pode esclarecer muito como este sistema funciona. Desta forma, a análise da arquitetura de uma rede cerebral e a conectividade de seus elementos ilumina algumas questões relacionadas com a integração das funções cerebrais e pode ajudar muito a construir uma ponte entre variações individuais de comportamento/cognição e variações nesta rede cerebral [Sporns, 2011].

Além do cérebro, outro sistema real modelado através das ferramentas da Teoria dos Grafos são as redes sociais, onde os vértices são pessoas ou grupos de pessoas, e as arestas são definidas por algum tipo de relacionamento entre elas [Newman, 2010], sejam pessoas que trabalham, ou estudam, ou realizam alguma atividade juntas, são amigas ou apenas conhecidos. É importante ressaltar que o tipo de interação que definirá as arestas entre os vértices dependerá do tipo de questão que se deseja investigar numa dada rede.

Os estudos que envolvem redes sociais datam desde muito antes de o termo se tornar conhecido através de ambientes computacionais como Facebook e Myspace. O trabalho do psiquiatra Jacob Moreno estimulou as observações das dinâmicas das interações sociais entre grupos de pessoas e foi reforçado com o lançamento do livro "Who Shall Survive" em 1934 [Moreno, 1934]. Os sociólogos, desde muito tempo, utilizam as redes e ferramentas da matemática e estatística em seus estudos sobre o que acontece no mundo real. Desta forma, instituíram um novo campo de estudos chamado sociometria, conhecido posteriormente como Análise de Redes Sociais [Newman, 2010].

Considerando estes dois sistemas reais, o indivíduo e seu cérebro, e a modelagem de ambos os sistemas como redes, supomos a possibilidade de que haja alguma correlação entre os padrões emergentes da topologia destas redes. Sendo assim, imagina-se que a participação do indivíduo numa rede social, cujas relações possam ser estabelecidas entre pessoas que realizam tarefas cognitivas específicas, por exemplo, possa oferecer dicas de como é o funcionamento do cérebro destes indivíduos durante a execução destas tarefas. Se o cérebro humano e as relações entre os seres humanos podem ser representados por uma estrutura de rede então talvez seja possível descobrirmos que correlações existem entre estes dois sistemas. Que tipo de influência a organização estrutural do cérebro pode ter nas relações pessoais deste indivíduo? E, 
vice-versa, que comportamentos sociais podem dar indícios sobre como funciona o cérebro daquela pessoa?

Este artigo está dividido da seguinte maneira: esta primeira parte introdutória, apresentando a motivação e a hipótese deste trabalho. A seção 2 faz um apanhado sobre a Teoria dos Grafos com suas propriedades principais, específicas para este estudo e métricas úteis para as análises de redes cerebrais e sociais. Na seção 3 encontra-se a descrição dos trabalhos correlatos que, através de experimentos em indivíduos, mostraram resultados que reforçaram as bases de nossa hipótese. A seção 4 é reservada para as conclusões deste trabalho e indicações a respeito de trabalhos futuros.

\section{Fundamentos das Redes}

O enfoque dos sociólogos, no uso da Teoria dos Grafos, esteve na análise de pequenas estruturas de rede e na identificação de elementos de rede conspícuos, tal como uma pessoa influente, por exemplo, [Wasserman e Faust, 1994]. Na última década os estudos sobre redes têm se expandido e incluído descrições estatísticas, revelando semelhanças e diferenças em organizações locais e globais de redes que representam o mundo real social, biológico, tecnológico e outros domínios [Sporns, 2011]. Especificamente no domínio da educação, para promover estímulo a aprendizagem seja mapeando conceitos [Borges et al, 2003] ou outro trabalho que mediu o desempenho pessoal de alunos através da relação social que estabeleciam participando de diferentes disciplinas [Maia et al, 2010].

Um grafo é a representação de um sistema do mundo real composto por elementos interconectados. Vértices são os elementos fundamentais deste sistema e arestas entre tais vértices existirão se houver uma relação entre eles [Sporns, 2011].

\section{1 - Métricas Importantes para o Estudo das Redes}

Um conjunto de métricas úteis, denominadas centralidade, dizem quão importantes são os vértices ou arestas numa rede. Há uma grande variedade de medidas matemáticas para calcular a centralidade de um vértice. Um exemplo é a medida chamada centralidade de grau. $\mathrm{O}$ grau de um vértice de uma rede é o número de arestas ligadas a ele. Em uma rede social, por exemplo, é o número de amizades de um indivíduo. Os vértices com os maiores graus em uma rede são aqueles com o maior número de conexões, e, em geral desempenham papel importante no funcionamento do sistema. Em geral, pode-se pensar que uma pessoa, numa rede de conhecidos com muitas conexões internas, possa ser alguém com mais influência, com mais acesso a informação ou com mais prestígio que outros presentes na mesma rede [Newman, 2010].

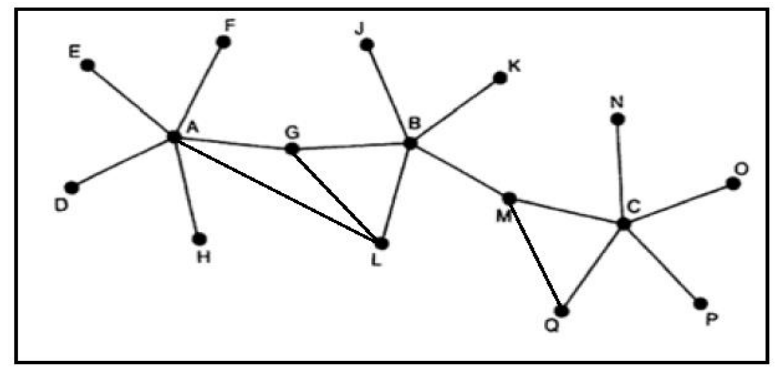

Figura 1: Exemplo de uma rede. 
Na Figura 1 têm-se o exemplo de uma rede, onde o número de vértices $\mathbf{n}$ é igual a 16 e m, o número de arestas, é igual a 18. Ainda sobre a centralidade de um vértice, esta pode ser observada de modo local, centralidade local calculando-se o número de conexões ao seu redor. A centralidade local de um vértice pode ser absoluta, resultado da soma das arestas diretamente conectadas a ele, ou relativa, ou seja, normalizada pelo número máximo de conexões possíveis. A saber, numa rede com n vértices, existem n-1 possibilidades de conexão, arestas, para cada vértice [Scott, 2000]. Arestas podem ser direcionadas, tendo um vértice origem e outro vértice destino, ou não direcionadas. Podem ser binárias (existem ou não) ou terem um peso associado a elas [Sporns, 2011].

A Matriz de adjacência ou matriz de conexão define a topologia do grafo sendo uma matriz onde os vértices são as linhas e as colunas estão relacionadas através da presença ou não de uma aresta entre tais vértices. São matrizes de 0's e 1's para grafos binários ou com números, representando os pesos das arestas. Vértices conectados por uma aresta são chamados de vizinhos. Da matriz de adjacência pode-se derivar o grau do vértice. Para grafos direcionados, têm-se o grau de entrada e grau de saída para o número total de arestas que entram e saem do vértice, respectivamente. Num grafo cujas arestas possuem peso, a soma dos pesos de todas as arestas de um vértice é um indicativo sobre a força deste vértice. A distribuição dos graus dos vértices de um grafo mostra o número de vértices com o mesmo grau e como se apresenta a variação destas quantidades num intervalo. [Sporns, 2011]

Outra medida importante é a densidade da rede. A densidade é um parâmetro relativo à configuração geral da rede, expressa a relação entre o número de arestas constituídas entre os vértices e o número total de arestas que poderiam ser constituídas [Scott, 2000].

A distância entre dois vértices é o menor caminho entre eles, ou seja, o caminho que contiver o menor número de arestas, também conhecido por caminho geodésico [Newman, 2010]. Por exemplo, dois amigos teriam distância geodésica $1 \mathrm{em}$ uma rede de amizade porque há uma única aresta conectando-os diretamente, enquanto o amigo de seu amigo teria distância 2 de você [Sporns, 2011]. Sendo assim, o diâmetro de um grafo é o maior caminho geodésico entre dois vértices deste grafo. [Newman, 2010].

Todos os pares de distância podem ser representados por uma matriz de distâncias. A estrutura das matrizes de adjacência e de distâncias descrevem padrões de comunicação dentro da rede. A presença de uma aresta mostra uma comunicação direta entre dois vértices. Caminhos de vários tamanhos registram possíveis maneiras pelos quais sinais podem ser trocados indiretamente entre dois vértices. Caminhos muito extensos entre dois vértices deixam os sinais trocados entre eles mais suscetíveis a perdas e ruídos. [Sporns, 2011]. Portanto, a distância média de uma rede fornece informação sobre o nível de eficiência na comunicação global de uma rede.

A métrica conhecida como coeficiente de agrupamento pode informar para cada vértice a densidade das conexões entre os vizinhos deste vértice. Vizinhos densamente conectados, ou seja, que estão conectados entre si, formam um grupo em torno do vértice. Para [Gilvan e Newman, 2002] o coeficiente de agrupamento é a probabilidade de dois vértices que são vizinhos de um terceiro serem vizinhos entre si. Na linguagem das redes sociais, dois de seus amigos terão uma maior probabilidade de conhecerem um ao outro pelo fato de já serem conhecidos seus. 
A média desta medida para cada vértice informa o coeficiente de agrupamento médio para a rede [Watts e Strogatz, 1998]. Redes com alto grau de agrupamento são geralmente composta por comunidades locais ou módulos contendo vértices densamente conectados entre si, compartilhando características ou mesmas funções [Gilvan e Newman, 2002]. Estes módulos aparecem separados porém conectados uns aos outros por poucas arestas. Pois, o fato de dois vértices estarem conectados entre si não significa que pertençam a uma mesma comunidade ou ainda que eles tenham algum outro relacionamento em comum [Wig et al, 2011].

O balanço entre a densidade interna ao módulo e a densidade externa, entre os módulos, define a medida de modularidade. Na Figura 2, vemos subconjuntos de vértices, ou módulos, densos internamente, e a rede esboçada apresenta poucas conexões entre um módulo e outro.

Para as redes cerebrais, unidades neuronais ou regiões cerebrais que formam um grupo densamente conectado, espalham melhor a informação entre seus componentes e então constituem um sistema funcionalmente coerente do cérebro. Consequentemente, unidades neuronais que pertençam a módulos ou grupos diferentes, não compartilham a mesma informação e também desempenham funções diferentes uns dos outros. A identificação destes módulos nas redes cerebrais são um passo importante para a compreensão destes subsistemas [Sporns, 2011].

Outras medidas capturam propriedades globais da rede como um todo, [Sporns 2011] entende que tais medidas transcendem os limites dos módulos e revelam a Integração Global da rede. Uma destas medidas, comum para medir a integração em redes cerebrais é o comprimento do caminho mínimo, ou a distância média entre os vértices da rede como um todo, e pode ser calculado através da média da matriz de distâncias [Watts e Strogatz, 1998]. O caminho mínimo indica que, na média, cada vértice pode ser alcançado por outro através de um caminho com poucas arestas.

Isto ajuda na compreensão de uma outra característica, observada em diferentes tipos de redes, que é o efeito small world [Newman, 2010][Newman, 2004]. O experimento famoso de Stanley Milgram [Travers e Milgran, 1969] deu suporte a comprovação deste fenômeno, e mostrou que duas pessoas quaisquer estão, em média, conectadas por não mais que "seis graus de separação". Num grupo social relativamente grande, duas pessoas estão conectadas por um caminho curto, formado por conhecidos, ou seja, o mundo dos relacionamentos é um mundo pequeno [Sporns, 2011].

Watts e Strogatz [1998] elaboraram um modelo de rede aparentemente simples que explicaria o fenômeno small world, com base nos padrões de conectividade, e descobriram que estes padrões estavam presentes na maioria das redes que se formam no mundo real, redes naturais, sociais e tecnológicas. Os padrões de conectividade que devem ser observados para a verificação ou não deste efeito são o coeficiente de agrupamento e a distância média da rede. Se uma rede apresenta um alto coeficiente de agrupamento, comparada a uma rede gerada randomicamente, e também apresenta uma distância média pequena, que é uma característica similar a uma rede gerada randomicamente, então esta rede apresenta o efeito small world. [Sporns et al, 2004]. 


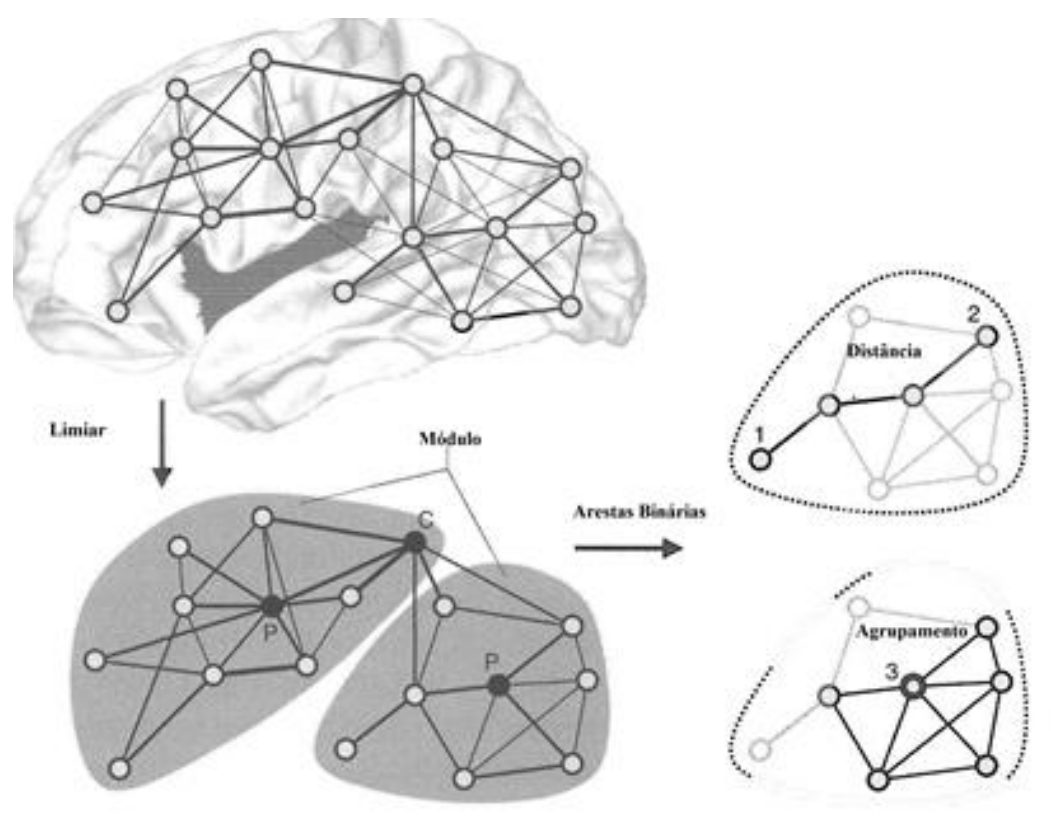

Figura 2 - Exemplo de modularização (extraído de Olaf Sporn (2011), Networks of The Brain, The MIT Press Cambridge, Massachusetts London, England, com alterações).

O exemplo na Figura 2 mostra uma rede com arestas não direcionadas e com pesos, antes (topo) e depois (abaixo) quando aplicado um limiar para a remoção de conexões fracas. A rede consiste em dois módulos, ligados por um vértice, que é um hub ("C") e cada módulo contém seu hub regional ("P"). Diagramas da direita mostram os módulos após as conexões terem sido binarizadas, ilustram um caminho geodésico ou distância ( 3 passos) entre os vértices 1 e 2 e o agrupamento ao redor do vértice 3 . $O$ nó 3 tem 5 vizinhos e estes vizinhos tem 5 das 10 possíveis arestas entre eles e então o coeficiente de agrupamento é 0,5 .

No entanto, para se ter uma ideia real sobre a integração de uma rede, o valor do caminho mínimo deve ser associado a outra medida chamada eficiência global. Em [Latora e Marchiori, 2001] esta medida é computada pela média da inversa da matriz de distância. Uma rede completamente conectada tem máxima eficiência pois a distância de cada vértice a outro vértice é de apenas uma aresta.

Uma rede com comprimento do caminho mínimo baixo ou com uma alta eficiência indica que pares de vértices, na média possuem uma distância pequena para comunicarem entre si e podem ser alcançados em poucos passos. Para redes cerebrais, por exemplo, alta eficiência e comprimento do caminho mínimo pequeno indica que seus vértices interagem mais diretamente e há alta integração funcional [Sporns, 2011].

Os vértices e arestas numa rede no mundo real possuem impacto com intensidade diferentes no funcionamento da rede. Alguns vértices são mais essenciais, ou mais influentes, que outros. Vértices importantes normalmente são mais densamente conectados que os demais, facilitando os processos de integração global e fazendo um papel de compensação quando a rede sofre um dano. Estes vértices são chamados de hubs. Para definir se um vértice é um hub, leva-se em consideração alguns critérios: seu grau, participação na conectividade entre módulos e outras medidas de centralidade. Em redes cuja distribuição de grau é não homogênea, os vértices com alto valor desta 
medida são frequentemente essenciais para a manutenção da conectividade da rede [Sporns, 2011].

No caso das redes sociais, os hubs atuam particularmente no fluxo da informação e na capacidade de resistência da rede como um todo, pois se falharem, ou seja, se o determinado site, que tem o papel de $h u b$, deixa de estar ativo, ou "sai do ar", ou uma certa pessoa, com esta mesma característica, deixa de fazer parte da rede, o fluxo de informação através destes vértices é interrompido e uma grande "quebra" entre conexões é esperada [Newman, 2010].

\section{3 - Indicativos da Validade da Hipótese}

Após relacionar os principais conceitos a respeito da Teoria dos Grafos que são de interesse especial para os estudos das redes cerebrais e das redes sociais, esta seção traz os trabalhos que contribuem para estruturar a hipótese deste artigo, focalizando nos estudos que trazem resultados sobre as possíveis relações entre as configurações das redes cerebrais e as respostas registradas em experimentos com seres humanos.

Nas redes cerebrais, os vértices representam elementos neurais como células, população de células ou regiões do cérebro. Arestas representam conexões entre vértices, como sinapses anatômicas ou caminhos entre regiões cerebrais. No entanto, as técnicas de observação existentes não são capazes de mapear um grande número de neurônios e suas relações sinápticas. Técnicas não invasivas utilizadas hoje cobrem regiões do cérebro registrando sinais originários de comunidades neurais. Sendo assim, a definição de vértice envolve uma divisão anatômica do cérebro em regiões coerentes, com base em dados histológicos e de imagem. Estudos neurocientíficos em humanos e animais têm dado evidências de que o cérebro é composto de áreas discretas e dissociáveis, e que estas áreas do cérebro exibem propriedades únicas que permitem diferenciá-las umas das outras [Sporns, 2011].

No entanto, a ciência das redes cerebrais possui um grande desafio no sentido de criar um mapa cerebral que permita identificar cada área individual do córtex. $\mathrm{O}$ trabalho de [Wig et al, 2011] traz um esclarecimento sobre algumas técnicas que devem ser utilizadas, de acordo com o objeto em estudo, para a definição de tais áreas de interesse e a consequente formalização sobre que componentes cerebrais serão representados pelos vértices e que tipo de relacionamento entre estes vértices definirão as arestas desta rede. As diferenças entre as técnicas adotadas refletem nos diferentes resultados que podem ser obtidos, tanto qualitativos quanto quantitativos a respeito da anatomia e funcionalidade de uma rede cerebral.

Estudos recentes têm mostrado que as ligações funcionais da rede cerebral estão organizadas de uma maneira altamente eficiente, na forma de small worlds, o que indica um elevado nível de agrupamento na vizinhança local de alguns vértices, que, em conjunto com um valor pequeno para a distância média entre as conexões desta rede, garantem um alto nível de eficiência da comunicação global dentro da rede como um todo. [Watts e Strogatz, 1998; Latora e Marchiori, 2001].

Em [Supekar et al, 2009] analisaram redes cerebrais construídas com base numa conectividade funcional relativas a crianças e jovens adultos. Propriedades como distância, coeficiente de agrupamento, análise da conectividade local foram medidas e revelaram que a organização cerebral dos participantes era similar a uma organização small world num nível global, sendo que a conectividade interna em diferentes regiões 
cerebrais foram diferentes para as faixas etárias analisadas. Este estudo demonstrou que a análise da conectividade das redes cerebrais pode esclarecer princípios básicos a respeito da maturação cerebral, permitindo o embasamento de outros estudos sobre características das estruturas da rede cerebral que podem indicar alguma desordem neural como o autismo, por exemplo. Assim como o trabalho de [Basset et al, 2009] que analisou redes cerebrais cujos sinais foram registrados durante a execução de tarefas para medir a performance da memória de trabalho em indivíduos saudáveis e em indivíduos com esquizoferenia.

Ainda sobre a medição da conectividade do cérebro através do mapeamento de uma rede cerebral, [Hampson et al, 2006] já haviam investigado a conectividade funcional entre duas áreas específicas do cérebro durante a execução de tarefas que exigiam trabalhar a memória e também durante um período onde os indivíduos participantes permaneceram em estado de relaxamento. Os dados resultantes da pesquisa reforçaram a possibilidade de que diferenças individuais na força da conexão entre estas regiões podem prever diferenças nas habilidades cognitivas importantes nas tarefas que exigem memória.

O trabalho de [Van den Heuvel , 2009b] examinou a relação entre a organização da rede cerebral e a inteligência. Através de uma comparação utilizando índices resultantes de medidas como o coeficiente de agrupamento e a distância média da rede, fez correlações entre estes e resultados de testes de QI. Apesar de que os participantes estivessem num estado de relaxamento e não executando uma tarefa cognitiva específica que pudesse ser medida dentro de uma escala de QI, os autores apresentam seus dados e os padrões de conectividade cerebral encontrados no intuito de fornecer algum tipo de indício de correlação com um desempenho cognitivo enquanto os indivíduos estiverem realizando tarefas. A conclusão do estudo indica que a característica de apresentar curtas distâncias médias é fundamental para o processamento eficiente e integração global de informação na rede cerebral e estão associadas à performance intelectual dos indivíduos.

As pesquisas em [Li et al 2009] também trazem a hipótese de que a organização estrutural do cérebro está associada aos diferentes resultados para os testes de inteligência realizados, e que há uma correspondência entre transferência eficiente de informação intracerebral de um indivíduo e suas respostas cognitivas. Para este estudo, foram construídas redes com arestas binárias e redes com arestas utilizando pesos referentes a cada um dos indivíduos participantes do experimento. Assim como em [Van den Heuvel, 2009b], chegou-se a conclusão de que os indivíduos que apresentaram maiores índices de inteligência nos testes de QI eram os mesmos cuja estrutura da rede cerebral apresentaram a característica de curtas distância entre seus vértices e uma eficiência global, com promissor indicativo da presença do efeito small world, indicando uma eficiente transferência de informação no cérebro. Os resultados são consistentes tanto para redes com aresta binarias quanto para arestas com pesos.

\section{Conclusões}

A neurociência têm adotado uma abordagem baseada em rede como uma lente sob a qual pode examinar a estrutura e o funcionamento do cérebro [Wig et al, 2011]. Através de cuidadosas medidas a respeito das relações entre as estruturas cerebrais têm-se chegado ao conceito de que o cérebro é uma grande rede, caracterizada por um emaranhado de conexões e relacionamentos. Visualizando o cérebro como um conjunto 
de elementos em constante interação e cuja organização é inerentemente complexa deve facilitar o nosso entendimento sobre sua arquitetura, suas funções e sobre comportamentos emergentes, seja em indivíduos saudáveis ou não.

Através de estudos teóricos e experimentos com seres humanos, muito já se tem visto sobre a existência de padrões emergentes das conexões funcionais das redes cerebrais associados a diferentes respostas cognitivas, sugerindo que o desempenho intelectual humano esteja relacionado à forma como o nosso cérebro integra informações entre suas várias regiões topológicas.

Assumindo a hipótese que que as redes cerebrais seguem uma estrutura de grafos análoga a das redes sociais e, planejou-se a realização futura de um experimento envolvendo alunos com diagnóstico de dislexia e outros sem o diagnóstico. Os indivíduos participariam de uma aula ministrada num ambiente virtual onde, através de um chat, se comunicariam com os outros alunos e com o professor. O texto oriundo destas conversas seriam analisados e gerariam grafos das relações estabelecidas entre os participantes e grafos individuais através do uso de suas palavras [Mota, et al, 2014]. O grafos gerados e as métricas empregadas sobre os mesmos auxiliariam na busca de pistas para o entendimento sobre o funcionamento cerebral e sobre os mecanismos que envolvem a aprendizagem.

\section{Referências}

Bassett DS, Bullmore ET, Meyer-Lindenberg A, Apud JA, Weinberger DR, et al. (2009). Cognitive fitness of cost-efficient brain functional networks. Proc Natl Acad Sci USA 1 06:1 1 747-1 1752.

Barabasi AL, Albert R. (1999). Emergence of scaling in random networks. Science 286:509-5 12.

Boorman ED, O'Shea J, Sebastian C, Rushworth MFS, Johansen-Berg H. (2007). Individual differences in white-matter microstructure reflect variation in functional connectivity during choice. Curr Bioi 17: 1426--1 431 .

Borges, FF, Dorça,F , Queiroz, B, Fernandes, M. A. ,Lopes, C.L., Teymeny, A.A, Ramos,A, (2003), Uma Ferramenta de Autoria para um Sistema Educacional Apoiado na Web, Anais do XIV Simpósio Brasileiro de Informática na Educação NCE - IM/UFRJ

Friston, KJ, (1993). Functional connectivity: The principal-component analysis of large (PET) data sets. J Cereb Blood Flow Metab 13 : 5-14.

Friston KJ. (1994). Functional and effective connectivity in neuroimaging: A synthesis. Hum Brain Mapp 2: 56-78.

Girvan M, Newman, M.E.J., (2002), Community structure in social and biological Networks. Proc Natl A cad Sci USA 99: 7821-7826.

Hampson M, Driesen NR, Skudlarski P, Gore JC, Constable RT. (2006). Brain connectivity related to working memory performance. J Neurosci 26: 13338-13343.

Li Y, Liu Y, Li J, Qin W, Li K, et al. (2009) Brain Anatomical Network and Intelligence. PLoS Comput Biol 5(5): e1000395. doi:10.1371/journal.pcbi.1000395 
Maia, RF, Spina, EM, Shimizu,SS (2010), Sistema de Previsão de Desempenho de Alunos para Auxilio a Aprendizagem e Avaliação de Disciplinas. Anais do XXI Simpósio Brasileiro de Informática na Educação - UFPB- Paraíba

Mota, N.B., Furtado, R., Maia, P.P.C., Copelli, M. \& Ribeiro, (2014) S. Graph analysis of dream reports is especially informative about psychosis. Sci. Rep. 4, 3691; DOI:10.1038/srep03691

Moreno, J.L. (1934), Who Shall Survive? Beacon House, Beacon, NY.

Newman, M. E. J., (2004), Coauthorship Networks and Patterns of Scientific Collaboration. Proceedings of the National Academy of Sciences of the United States of America 101.Suppl 1, páginas: 5200-5205.

Newman, M.E.J. (2010), Networks An Introduction, Oxford University Press Inc., New York, EUA.

Scott, J., (2000), Social Network Analysis - A Handbook, Sage Publication

Sporns O, Tononi G, EdelmanGM (2000) Connectivity and complexity: the relationship between neuroanatomy and brain dynamics. Neural Netw 13:909 -922.

Sporns O, Chialvo DR, Kaiser M, Hilgetag CC (2004) Organization, developmet and function of complex brain networks. Trends Cogn Sci 8:418-425.

Sporns 0. 2006. Small-world connectivity, motif composition, and complexity of fractal neuronal connections. Biosystems 85: 55-64

Sporn,Olaf (2011), Networks of The Brain, The MIT Press Cambridge, Massachusetts London, England

Supekar K, Musen M, Menon V (2009) Development of Large-Scale Functional Brain Networks in Children. PLoS Biol 7(7): e1000157. doi:10.1371/journal.pbio.1000157

Travers J, Milgram S. (1969). An experimental study of the small world problem. Sociometry, 32: 425-443.

van den Heuvel, MP, Mandl, RCW, Kahn, RS, Hulshoff, Pol HE, (2009a), Functionally linked resting-state networks reflect the underlying structural connectivity architecture of the human brain. Hum Brain Mapp. Advance online publication. Retrieved May 13, 2009. doi:10.1002/hbm.20737.

van den Heuvel, MP, Starn CJ, Kahn RS, Hulshoff Pol H E . (2009b). Efficiency of functional brain networks and intellectual performance. J Neurosci 29: 761 9-7624.

Wasserman, Stanley; Faust, Katherine (1994) Social network analysis: Methods and applications. Structural analysis in the social sciences. New York, NY, US: Cambridge University Press. xxxi 825 pp.

Watts, D. J. and Strogatz, S. H., (1998), Collective dynamics of 'small-world' networks, Nature 393, 440-442.

Wig,GS, Schlaggar,BL e. Petersen ES, (2011), Concepts and principles in the analysis of brain networks, doi: 10.1111/j.1749-6632.2010.05947.x126 Ann. N.Y. Acad. Sci. 1224, 126-146 New York Academy of Sciences. 